\title{
Medical Device Failure or Malfunction
}

National Cancer Institute

\section{Source}

National Cancer Institute. Medical Device Failure or Malfunction. NCI Thesaurus. Code C62996.

An adverse event and reportable event where a medical device may have caused or contributed to a death or serious injury. 